\title{
Nephroblastomatosis: imaging challenges
}

\author{
J. Damien Grattan-Smith
}

Received: 12 January 2011 / Accepted: 17 January 2011

(C) Springer-Verlag 2011

Nephroblastomatosis is a rare pre-neoplastic lesion defined as the presence of multiple nephrogenic rests. These are abnormal foci of persistent embryonal cells beyond 36 weeks' gestation that are thought to be precursors for the development of Wilms tumor. Nephrogenic rests are seen in approximately $1 \%$ of pediatric autopsy series. Nephrogenic rests and nephroblastomatosis represent a spectrum of the same disease and the distinction between the two is somewhat arbitrary. There are no formal quantitative criteria that determine how many nephrogenic rests constitute nephroblastomatosis. The nephrogenic rests are divided into four categories in the pathology literature: perilobar, intralobar, combined and diffuse, depending on their location within the renal parenchyma. On imaging, nephroblastomatosis is best classified as multifocal or diffuse. Virtually all cases of bilateral Wilms tumor have associated multifocal nephroblastomatosis.

Advances in imaging have increased our ability to detect small renal lesions in situ and, therefore, have played an increasingly significant role in the preoperative diagnosis and postoperative management of nephroblastomatosis and Wilms tumor. Imaging studies are essential in the diagnosis of these lesions and their evaluation over time to detect transformation into Wilms tumor. State-ofthe-art imaging with contrast-enhanced CT and MRI

Disclaimer Dr. Grattan-Smith has no financial interests, investigational or off-label uses to disclose.

J. D. Grattan-Smith $(\bowtie)$

Children's Healthcare of Atlanta at Scottish Rite,

Atlanta, GA 30342, USA

e-mail: damien.grattan-smith@choa.org routinely detects lesions as small as $5 \mathrm{~mm}$ in diameter. Nephroblastomatosis is considered a predisposing condition to Wilms tumor development both in the ipsilateral and contralateral kidney. Identifying and distinguishing between nephroblastomatosis and Wilms tumor is critical because they imply distinct prognoses and treatment decisions. Differentiation between Wilms tumor and nephroblastomatosis is based on histological evaluation of biopsy combined with imaging studies. Fine-needle aspiration cytology is of limited value in the assessment of these lesions, as cytology is not able to distinguish nephroblastomatosis from Wilms tumor.

The anatomical location of nephrogenic rests varies as well as the pattern of their distribution. Importantly, multifocal nephroblastomatosis has a stronger association with Wilms tumor than the diffuse type. The diffusely hyperplastic perilobar nephroblastomatosis has a characteristic appearance that is identified as peripheral confluent masses distorting and compressing the kidney with a thick rind of uniform abnormal tissue. On CT and MR imaging, both types appear similar to the renal cortex on nonenhanced scans. After contrast administration, NRs become markedly hypodense on CT and hypointense on MRI in comparison with the intensely enhancing renal parenchyma. Although there are no absolute imaging criteria to differentiate nephrogenic rests and nephroblastomatosis from Wilms tumor, nephrogenic rests and nephroblastomatosis can be differentiated from Wilms tumor in the same kidney, as Wilms tumors tend to be larger and more heterogeneous and demonstrate some contrast enhancement.

Gadolinium-enhanced T1-weighted MRI is currently touted as the optimal imaging approach for detecting nephrogenic tissue, although US and CT are commonly 
used. Using US, nephrogenic rests are usually homogeneously hypoechoic or isoechoic relative to normal kidney, but occasionally present as hyperechoic masses. Highresolution US can detect small nephrogenic rests but is reported to have poorer overall sensitivity than $\mathrm{CT}$ or MR imaging. Although US might miss small nephrogenic rests, it is excellent in recognizing larger lesions and the more extensive lesions of diffuse nephroblastomatosis. In these cases, US can readily depict the focal masses or the characteristic thick hypoechoic rind encasing the normal renal parenchyma. Diffuse nephroblastomatosis is usually hypoechoic and the multifocal type often isoechoic when compared to the normal renal parenchyma. US is the primary modality used for screening and surveillance because it is simple to perform, does not require sedation or intravenous contrast medium, uses no ionizing radiation and is relatively inexpensive.

Studies comparing the various imaging modalities have judged contrast-assisted MR imaging as superior for the detection of nephrogenic rests and nephroblastomatosis, although the advent of multidetector CT with multiplanar reformations might challenge MR in the detection of these lesions. Contrast enhancement with MR or CT increases the conspicuity of nephrogenic tissue. The nephrogenic rests do not enhance as vigorously as normal kidney and become contrasted against the background of normal enhancing renal parenchyma. T1-weighted, proton density and T2weighted MR imaging do not readily depict nephrogenic rests because the lesions are variable in intensity and can be difficult to distinguish from the surrounding renal parenchyma. Small lesions might appear more conspicuous with MR than with US or CT, especially when using highresolution T2 and contrast-enhanced STIR imaging.
Imaging plays an essential role in the pre- and posttreatment phases of afflicted patients. The pre-treatment roles of imaging include:

(1) Identification of nephrogenic rests in the kidney contralateral to a Wilms tumor,

(2) differentiation of diffuse nephroblastomatosis from Wilms tumor, and

(3) screening of patients at risk for nephroblastomatosis or Wilms tumor.

Gadolinium-enhanced T1- weighted MR imaging is often used to monitor regression or progression of nephrogenic tissue. Using this modality, involution is suggested by a reduction in size of the nephrogenic tissue, as well as increased hypointensity relative to the original lesion. Presumably, this change is a consequence of increased fibrosis and subsequent devascularization of the nephrogenic rest. The loss of vascularity results in diminished uptake of contrast media by the sclerosing rest. For practical purposes, increasing size of these lesions is the indicator used to screen for the development of Wilms tumor.

Therapeutic management of nephroblastomatosis both with and without Wilms tumor is controversial. It is not known to what extent chemotherapy for Wilms tumor ablates or deactivates associated nephrogenic rests. Preoperative chemotherapy is sometimes used to preserve normal parenchyma and to prevent transformation of nephroblastomatosis into Wilms tumor. Chemotherapy might decrease the incidence of developing Wilms tumor. Surgery is not recommended as the initial treatment for nephroblastomatosis and should be avoided as long as possible because of the increased chance of a metachronous tumor developing in the contralateral kidney. 